\title{
Fifty brilliant years
}

\author{
The first demonstration of the laser has not only led to a myriad of commercial applications, but fifty \\ years on basic research continues to rejuvenate the fundamental physics of the laser.
}

Fifty years ago, on 16 May 1960, the first laser was demonstrated at Hughes Research Laboratories by Theodore Maiman ${ }^{1}$. On the occasion of this anniversary, this issue of Nature Materials highlights the importance of the laser through a number of editorial projects: we present a series of interviews, where four leading scientists comment on the importance of the laser. Our first interview is with Charles Townes, who pioneered both the maser and the laser ${ }^{2}$. He talks about the developments that led to the realization of the maser, and based on that, the laser. This is followed by an interview with Herbert Kroemer, an inventor of the double-heterostructure semiconductor laser. His work led to the first demonstration of room-temperature solid-state lasers, and consequently has enabled technological developments such as compact-disk drives and optical telecommunications ${ }^{3}$. Our interview with Federico Capasso then highlights the developments of advanced semiconductor lasers, notably the quantum cascade laser that was developed in his $\mathrm{lab}^{4}$. Last but not least, the interview with Gerhard Materlik, CEO of the Diamond Light Source in the UK, considers the laser from the perspective of a researcher working with synchrotrons and free-electron lasers ${ }^{5}$.

In addition, Nature Materials has coproduced the Nature Milestones: Photons collection ${ }^{6}$. Following the format for such collections, this supplement features a series of highlights on milestones in research related to photons, ranging from fundamental principles such as Maxwell's equations to more applied topics such as, of course, the laser, optical fibres, plasmonics and many more. Furthermore, we reprint some selected historic papers, including Maiman's first laser paper, which was published in Nature in August 1960. This collection is distributed to all our print subscribers and is free to access online for registered users of nature.com until the end of this month.

Looking at the history of research into photons and their applications, the impact of the laser on many areas of research is very evident, making it one of the greatest achievements in modern science. Fifty years after its first demonstration, the use of the laser has become widespread not only in commercial applications but also as a versatile research tool in areas ranging from

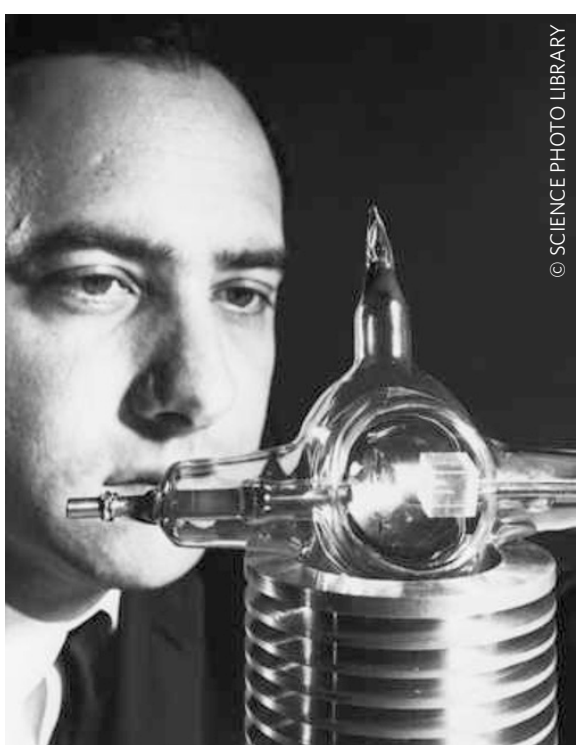

Theodore Maiman, the maker of the first laser.

fundamental physics to chemistry, biology and medical research.

However, in comparison with some of the other great inventions in science and technology, fundamental laser research continues to be rejuvenated. More than fifty years after the demonstration of the first transistor in 1947, transistors are more powerful, much smaller and are found in their millions on a single computer chip, yet their fundamental design principles remain unchanged. Fifty years after Karl Benz built the first modern automobile in 1885, cars were mass-fabricated and became ubiquitous in modern life, yet today their basic shape and function remains largely the same.

Lasers on the other hand have demonstrated a versatility and capability for constant innovation that surpasses that of many other main scientific discoveries. A beautiful case in point mentioned by Federico Capasso ${ }^{4}$ is the concept of matter waves, and consequently the realization of matter lasers in Bose-Einstein condensates. Another example of the laser concept transferred to different types of waves is phonon lasers, which are based on the coherent emission of phonons rather than photons ${ }^{7}$.

At the same time, the optical laser continues to push the extremes of physics.
The realization of lasers emitting intense, attosecond-long pulses enables the observation of atomic-scale electron motion. And researchers are already venturing towards zeptosecond pulses, whose duration is shorter than the oscillation period of an electron's wavefunction in an atom. Such lasers could open entirely new possibilities in the imaging of electron motions ${ }^{8}$.

This type of diversity in laser physics is bound to continue. For sure, new types of lasers will continue to emerge - also in the spirit of laser pioneer Arthur Schawlow's jocular notion that anything will lase if pumped hard enough. At the same time, the pathways to the generation of laser radiation may not always follow conventional wisdom. Lasers without population inversion are one example where modern developments have revised our understanding of the fundamental operation principles of lasers. Similarly, new developments will continue to push extremes in laser parameters. Be it the high-power lasers that drive research into nuclear fusion at the National Ignition Facility at the Lawrence Livermore National Laboratory in California. Or nanoscale lasers based on the interaction with surface plasmons, so-called spasers ${ }^{9}$, which challenge another dogma in laser science, namely that lasers require resonant cavities larger than roughly half the wavelength of the laser light. Such spasers could lead to new functionality on photonic chips.

Fifty years after the first laser, research in lasers still takes unusual turns. Not only does the development and enhancement of conventional laser sources continue in academic and industrial research labs, but the very basic laser concept is constantly renewed and revived. Unlike the story of the automobile, or indeed the transistor, the story of the laser remains very much an open book and may still take a few unexpected yet profound new twists.

\footnotetext{
References

1. Maiman, T. H. Nature 187, 493-494 (1960).

2. Nature Mater. 9, 371-372 (2010).

. Nature Mater. 9, 372-373 (2010).

. Nature Mater. 9, 374-375 (2010).

. Nature Mater. 9, 375-377 (2010).

6. http://www.nature.com/milestones/photons/

Vahala, K. et al. Nature Phys. 5, 682-686 (2009)

. http://go.nature.com/sqVGtj

9. Bergman, D. J. \& Stockman, M. I. Phys. Rev. Lett. 90, 027402 (2003).
} 